\title{
Expansion dynamics of laser produced plasma
}

\author{
B. Doggett and J. G. Lunneya) \\ School of Physics, Trinity College Dublin, Dublin 2, Ireland
}

(Received 13 January 2011; accepted 19 February 2011; published online 4 May 2011)

\begin{abstract}
We consider the applicability of the isentropic, adiabatic gas dynamical model of plume expansion for laser ablation in vacuum. We show that the model can be applied to ionized plumes and estimate the upper electron temperature limit on the applicability of the isentropic approximation. The model predictions are compared with Langmuir ion probe measurements and deposition profiles obtained for excimer laser ablation of silver. (C) 2011 American Institute of Physics. [doi:10.1063/1.3572260]
\end{abstract}

\section{INTRODUCTION}

The removal of material from a surface by irradiation with a high intensity pulsed laser beam leads to a cloud of ablated material moving rapidly away from the target surface. Near the ablation threshold the ablation cloud consists of neutrals, ions, and electrons. The plume shape and the ion velocity distribution are important for applications of laser ablation in mass spectrometry, ${ }^{1}$ laser induced breakdown spectroscopy (LIBS), and in pulsed laser deposition (PLD) of thin films ${ }^{2}$ and nanoparticle production. ${ }^{3}$ For example, in PLD the thickness distribution during film deposition on a substrate is determined by the plume shape that has evolved during the expansion from the target surface to the substrate.

The initial heating of the target leads to a strong evaporative ejection of material. Since the heating is extremely fast, surface temperatures close to the thermodynamic critical temperature can be reached. The laser light is partly absorbed by the plume, and partly transmitted through the plume to the evaporating surface. The plume is heated by various absorption processes and the resultant heating drives the expansion away from the target. A simple description is that the expansion of the plume is driven in a dynamical equilibrium, in such a way that the absorption of laser light provides the kinetic energy for the expansion. For typical nanosecond-PLD conditions with a fluence from 1-10 $\mathrm{J} \mathrm{cm}{ }^{-2}$ over a spot of $0.01-0.1 \mathrm{~cm}^{2}$ the ablation yield ranges from $10^{15}-10^{16}$ atoms per pulse. ${ }^{4}$ At the end of the laser pulse, the ablated material exists as a layer of dense gas up to $100 \mu \mathrm{m}$ thickness and is expanding away from the surface.

Figure 1(a) shows a schematic illustration of the expanding ablation plume. It begins as a thin layer of vapor/plasma on the target with lateral radii $X_{0}$ and $Y_{0}$ and thickness $Z_{0}$ which is typically $10-100 \mu \mathrm{m}$. At a later stage the ellipsoidal plume has radii $X(t), Y(t)$ and $Z(t)$. After the end of the laser pulse there is little further energy or mass input to the plume, and the expansion can be considered as adiabatic. Figure 1(b) shows a schematic of the probe positioning to determine the angular distribution of the ablated particles. The behavior of the plasma expansion depends primarily on the initial plume dimensions and background gas pressure. For low gas pressures $\left(<10^{-3}\right.$ mbar $)$ the gas has little influence on the

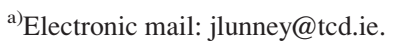

expansion, which is essentially inertial after an initial acceleration phase of a few mm. After this phase, the expansion velocity of the plume front in any direction remains nearly constant. The plume expansion can be described using hydrodynamic models, by Monte Carlo simulations, or a combination of both. ${ }^{5}$ Any model of the plume expansion must account for at least three features:

(i) The strongly peaked expansion in the direction perpendicular to the surface.

(ii) Broadening of the angular distribution with decreasing laser spot size.

(iii) The effect whereby the lateral expansion is larger in the direction corresponding to the smaller initial ablation spot dimension.

The adiabatic expansion models of Anisimov et al. ${ }^{6}$ and Singh and Narayan ${ }^{7}$ have proved to be very useful for the understanding and interpretation of laser ablation experiments. Both models consider the adiabatic expansion of a small volume of hot gas into vacuum. The Anisimov model considers the expansion to be isentropic, while in the Singh and Narayan model it is isothermal. The dimensions of the volume of gas are chosen to correspond to the dimensions of the vaporized material at the time when evolution and heating of the ablated material is complete. For nanosecond (ns) ablation this time is approximated by the end of the laser pulse.

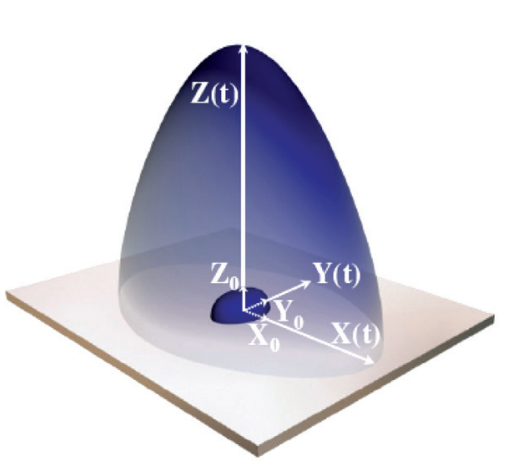

(a)

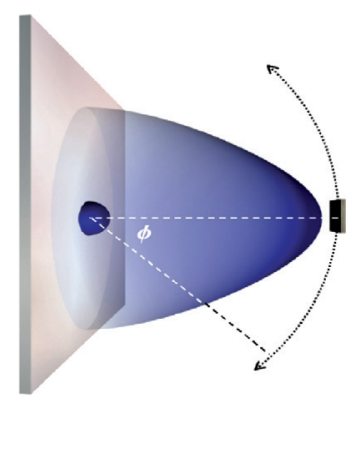

(b)
FIG. 1. (Color online) (a) Diagram showing the plume at the end of the laser pulse when the dimensions are $X_{0}, Y_{0}$, and $Z_{0}$, and after a time $t$ when the dimensions are $X(t), Y(t), Z(t)$. (b) A schematic of the probe positioning to determine the angular distribution of the ablated particles. 
The density gradients in the plasma drive the expansion which leads to supersonic flow. Since the gradient is greatest normal to the target, the acceleration of flow is greatest in the same direction resulting in the usual forward directed ablation plume. Considering the expression for the electronion energy equipartition given by Spitzer, ${ }^{8}$ it is clear that the electron-ion collision rate is sufficient to maintain the equality of temperature between the electrons and ions, i.e., $T_{e}=T_{i}=T$. Thus the plasma pressure will be

$$
P=n_{i}(Z+1) k T,
$$

where $n_{i}$ is the ion density, $Z$ is the mean ion charge, $k$ is the Boltzmann constant, and $T$ is the temperature. Since the Debye length is much smaller than the plasma dimension we can ignore the influence of charge separation on the expansion dynamics. Following the analysis of Attwood, ${ }^{9}$ it can be seen that there are two contributions to the gradient of plasma pressure. The first is due to the ion density gradient, which in each dimension is given by

$$
\frac{\partial P_{i}}{\partial x}=\gamma k T \frac{\partial n_{i}}{\partial x}
$$

where $\gamma$ is the ratio of specific heats. Secondly, the gradient of electron density gives rise to an ambipolar electric field:

$$
E=-\frac{1}{e n_{e}} \frac{\partial P_{e}}{\partial x}=-\frac{1}{e n_{e}} \gamma k T \frac{\partial n_{e}}{\partial x},
$$

which, of course, will act on the ions. The ion momentum equation is then given by

$$
m_{i} n_{i}\left[\frac{\partial}{\partial t}+v_{i} \frac{\partial}{\partial x}\right] v_{i}=-(Z+1) \gamma k T \frac{\partial n_{i}}{\partial x},
$$

where $m_{i}$ is the ion mass and $v_{i}$ is the ion flow velocity. This is the same starting point as used by Anisimov et al. in the analysis of the expansion of a small volume of hot vapor. Thus it is valid to use the models developed by Anisimov et al. and Singh and Narayan to describe the expansion of ionized laser ablation plumes.

However the following question remains: is the expansion isentropic or isothermal? Essentially we want to know if electron heat diffusion is sufficiently rapid to keep pace with the plasma expansion. Taking the Spitzer formula for the thermal conductivity of plasma it can be shown that heat will diffuse a distance, $s$, in time, $t$, given by

$$
s^{2}=\frac{9.3 \times 10^{19} T^{5 / 2}}{Z(Z+1) n_{i}} t
$$

where $T$ is in $\mathrm{eV}$ and $n_{i}$ in $\mathrm{cm}^{-3}$. The rate of plasma expansion is of the same order of the ion sound speed, $c_{s}$, and in one dimension, the size, $l$, of a freely expanding plasma after time, $t$, is approximately

$$
l=c_{s} t=9.79 \times 10^{5}\left(\frac{\gamma Z T}{m_{i}}\right)^{1 / 2} t .
$$

For as long as the expansion is approximately one-dimensional; $n_{i} l=n_{s} d$, where $n_{s}$ is the atom density in the target and $d$ is the ablation depth. Thus,

$$
n_{i}=\frac{n_{s} d}{l}
$$

Substituting for $n_{i}$ in Eq. (5) leads to

$$
s^{2}=\frac{9.3 \times 10^{19} T^{5 / 2}}{Z(Z+1) n_{s} d} \text { lt. }
$$

For an isentropic plasma expansion we require $s<l$, which from Eqs. (6) and (7) yield

$$
T^{2}<1.05 \times 10^{-14} \gamma^{1 / 2} Z^{3 / 2}(Z+1) n_{s} d m_{i}^{-1 / 2} .
$$

Taking $\gamma=5 / 3, Z=1, d=10^{-6} \mathrm{~cm}$, and for $\mathrm{Ag}$, $n_{s}=5.86 \times 10^{22} \mathrm{~cm}^{3}$ and $m_{i}=108$ we get

$$
T<12.4 \mathrm{eV} \text {. }
$$

Thus it would seem that for typical ns ablation conditions the plume expansion may be treated as isentropic if the plasma temperature is less than about $12 \mathrm{eV}$, which is normally the case.

Though the Anisimov expansion model was developed for the expansion of a neutral gas, we show it to be applicable to laser produced plasma (LPP), particularly when the electron temperature is below $12 \mathrm{eV}$, as it is in this work. ${ }^{10-12}$ In this paper the plume expansion under vacuum originating from such a dense, initial plume will be described and discussed. In order to make a direct comparison of the model and experiment, the following parameters need to be determined: the shape and velocity of the plume at various times after the ablating laser pulse and the spatial distribution of density and temperature. There is a wide range of experimental techniques that have been used to diagnose laser ablation plumes, and these have been reviewed by Amoruso et al. ${ }^{13}$ Broadly speaking, the primary diagnostic techniques can be divided into optical and electrical probe methods. In this paper we concentrate on results obtained using electrical probes. The technique of using a probe, (also known as a Langmuir probe) to measure the properties of a LPP was first introduced by Koopman ${ }^{14}$ and has become a popular technique. ${ }^{10-12,15}$ Essentially, a Langmuir probe consists of a small bare electrical conductor which is immersed in a plasma and can be biased with respect to the plasma potential. Typically the plasma expansion is supersonic, thus the plasma flow velocity is much greater that the Bohm velocity $u_{B}=\left(e T_{e} / m_{i}\right)^{1 / 2}$. For a negatively biased probe oriented normal to the plasma flow, the ion current will be dominated by the ion flux due to the flow. For large negative bias, the magnitude of the ion signal from a planar probe does not depend on the bias. When the ion velocity is determined by the ion time-of-flight (TOF) from the target to the probe, the ion density can be found from the magnitude of the ion signal. If the probe is biased positively a net electron current will be recorded. Starting at the plasma potential and changing the probe bias to more negative values leads to a reduction of the electron current as electrons of increasingly higher energy are unable to reach the probe. This region of the $I-V$ characteristic can be used to find the electron temperature. ${ }^{11,12}$ 


\section{ANISIMOV MODEL OF PLUME EXPANSION}

The model developed by Anisimov et al. treats the adiabatic expansion of a one-component vapor cloud into vacuum using a particular solution of the gas-dynamic equations, which applies when the flow is self-similar. It is assumed that the formation time of the vapor cloud is much less than its expansion time and that the focal spot of the laser has an elliptical shape with semiaxes $X_{0}$ and $Y_{0}$. The expansion is modeled as a triaxial gaseous semiellipsoid whose semiaxes are initially equal to $X_{0}, Y_{0}$, and $Z_{0} \approx c_{s} \tau_{p}$, where $\tau_{p}$ is the duration of the laser pulse, and $c_{s}$ is the sound speed in the vaporized material given by $c_{s}=[\gamma(\gamma-1) \varepsilon]^{1 / 2}$. Here $\varepsilon=E_{p} / M_{p}$ is the energy per unit mass, where $E_{p}$ is the thermal energy of the initial plume and $M_{p}$ is its mass. For a monatomic ideal gas $\gamma=5 / 3$. However for low temperature plasma the value is lower; Zeldovich and Raizer have suggested a value of 1.24 . $^{16}$ Previously values in the range of 1.1-1.4 have been found in the analysis of expansion of laser produced plasma. ${ }^{10,11}$ In this paper a value of 1.25 is used.

The gas dynamic equations are

$$
\begin{aligned}
& \frac{\partial \rho}{\partial t}+\operatorname{div}(\rho v)=0 \\
& \frac{\partial v}{\partial t}+(v \nabla) v+\frac{1}{\rho} \nabla p=0 \\
& \frac{\partial S}{\partial t}+v \nabla S=0
\end{aligned}
$$

where $\rho, p, v$, and $S$ are the density, pressure, velocity and entropy of the gas, respectively. It is assumed that the flow parameters are constant on ellipsoidal surfaces and the density and pressure profiles can be written as

$$
\begin{aligned}
& \rho(r, t)=\frac{M_{p}}{I_{1}(\gamma) X Y Z}\left[1-\frac{x^{2}}{X^{2}}-\frac{y^{2}}{Y^{2}}-\frac{z^{2}}{Z^{2}}\right]^{1 / \gamma-1}, \\
& p(r, t)=\frac{E_{p}}{I_{2}(\gamma) X Y Z}\left[\frac{X_{0} Y_{0} Z_{0}}{X Y Z}\right]^{\gamma-1}\left[1-\frac{x^{2}}{X^{2}}-\frac{y^{2}}{Y^{2}}-\frac{z^{2}}{Z^{2}}\right]^{\gamma / \gamma-1} .
\end{aligned}
$$

$I_{1}(\gamma)$ and $I_{2}(\gamma)$ are functions of the adiabatic index, and are defined in Ref. 6. The gas-dynamic equations can be reduced to a set of ordinary differential equations

$$
\begin{aligned}
\ddot{X} & =-\frac{\partial U}{\partial X}, \\
\ddot{Y} & =-\frac{\partial U}{\partial Y}, \\
\ddot{Z} & =-\frac{\partial U}{\partial Z}, \\
U & =\frac{5 \gamma-3}{\gamma-1} \frac{E_{p}}{M_{p}}\left[\frac{X_{0} Y_{0} Z_{0}}{X Y Z}\right]^{\gamma-1}
\end{aligned}
$$

with the initial conditions set as

$$
\begin{aligned}
& X(0)=X_{0}, Y(0)=Y_{0}, Z(0)=Z_{0}, \\
& \dot{X}(0)=\dot{Y}(0)=\dot{Z}(0)=0 .
\end{aligned}
$$

From Eq. (10), the mass flux at a point $\left(x_{p}, y_{p}, z_{p}\right)$ in the $z$ direction is

$$
F\left(z_{p}, t\right)=\rho\left(x_{p}, y_{p}, z_{p}, t\right) v_{z}\left(x_{p}, y_{p}, z_{p}, t\right),
$$

where $v_{z}\left(x_{p}, y_{p}, z_{p}, t\right)=\left(z_{p} / Z\right) \dot{Z}$. In the asymptotic limit of constant plume front velocity, the time $t_{f}$, at which the front reaches a hemispherical substrate of radius $R$ centered on the ablation spot and an angle $\phi$ in the $x$-z plane with respect to the normal, is

$$
t_{f}=\frac{R\left[1+k_{x}^{2} \tan ^{2} \phi\right]^{1 / 2}}{(d Z / d t)\left[1+\tan ^{2} \phi\right]^{1 / 2}} .
$$

The particle flux on to the hemispherical surface can be expressed in units of $t / t_{f}$ :

$F\left(\phi, \frac{t}{t_{f}}\right) \frac{d t}{t_{f}}=\frac{M_{p} k^{2}}{m I_{1}(\gamma) R^{2}}\left(\frac{1+\tan ^{2} \phi}{1+k_{x}^{2} \tan ^{2} \phi}\right)^{3 / 2} \times \frac{t_{f}^{4}}{t^{4}}\left[1-\frac{t_{f}^{2}}{t^{2}}\right]^{1 / \gamma-1} \frac{d t}{t_{f}}$

$k_{x}=Z / X$ is the ratio of the semiaxes in the $x-z$ plane and characterizes the forward-peaking of the plume. A similar expression describes the angular variation of particle flux in the $y-z$ plane. The expression for plume temperature from the model is

$$
T(z, t)=\frac{\varepsilon(5 \gamma-3)(\gamma-1)}{2 \gamma}\left(\frac{X_{0} Y_{0} Z_{0}}{X Y Z}\right)^{\gamma-1}\left[1-\left(\frac{z}{Z}\right)^{2}\right] .
$$

Figure 2(a) shows the radial variation of the temperature, density and flow velocity within the plume according to the

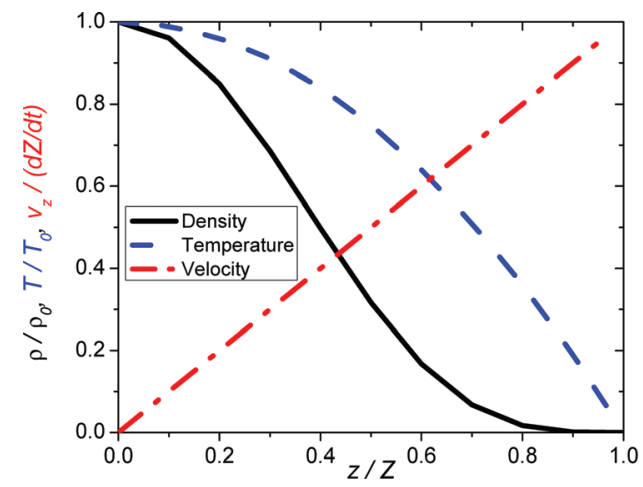

(a)

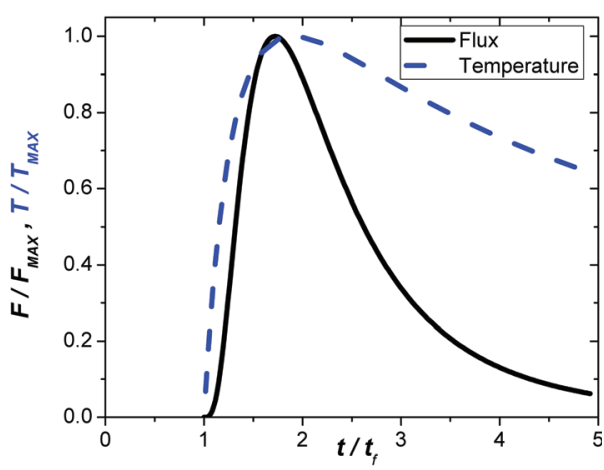

(b)
FIG. 2. (Color online) (a) The spatial variation of the density, temperature, and velocity along the $z$-axis. $Z$ is the plume front. (b) Temporal variation of the particle flux [Eq. (15)] and the temperature [Eq. (16)] variation at a position in front of the target. The time axis is normalized to the front arrival time $\left(t_{f}\right)$ at that position. 


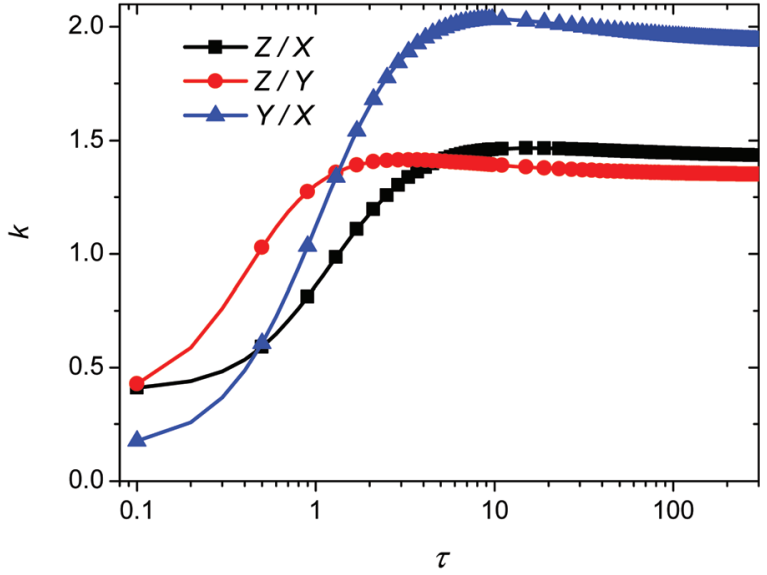

FIG. 3. (Color online) Temporal evolution of various plume aspect ratios for $X_{0}: Y_{0}: Z_{0}=1: 0.4: 0.13$ and $\gamma=1.25$.

isentropic model of plume expansion. The temperature at the plume boundary is zero; this is a consequence of the assumption of isentropic expansion where heat conduction is not sufficiently fast to carry energy through the expanding plume. Figure 2(b) shows the temporal variation of the particle flux [Eq. (15)] and plume temperature [Eq. (16)] at a position in front of the target. The time axis is normalized to the time $\left(t_{f}\right)$ taken for the plume to reach that position. Figure 3 shows the predicted variation of the plume aspect ratios as a function of the dimensionless time $\tau$, defined as follows:

$$
\tau=\frac{t}{X_{0}}\left((5 \gamma-3) \frac{E_{p}}{M_{p}}\right)^{1 / 2} .
$$

For this simulation the initial aspect ratios are $X_{0}: Y_{0}$ : $Z_{0}=1: 0.4: 0.13$ and $\gamma=1.25$. It can be seen from Fig. 3, that for $\tau \approx 1$, the $Y$ dimension is greater than the $X$ dimension even though $Y_{0}<X_{0}$. This apparent rotation of the semiaxis of the ellipsoidal plume arises for noncircular ablation spots, since there is a higher pressure gradient and higher plasma acceleration in the direction where the initial plume dimension is smaller. For a rectangular or elliptical beam spot it means that the plume evolves in such a way that the major axis lies $90^{\circ}$ to that of the beam spot. Figure 3 also shows that the acceleration phase lasts until $\tau \sim 5$, after this time the plume expands with constant velocity.

The angular dependence of the number of particles arriving per unit area normal to the flow $f(\phi)$ can be found by integrating the flux [Eq. (15)], from the arrival time, $t_{f}$, to infinity. The analytical expression for $f(\phi) / f(0)$, describing the deposition per unit area on a hemisphere in the plane $x-z$ will be

$$
\frac{f(\phi)}{f(0)}=\left(\frac{1+\tan ^{2} \phi}{\left[1+\left(k_{x}^{2}\right) \tan ^{2} \phi\right]}\right)^{3 / 2} .
$$

\section{EXPERIMENT}

To create the plasma, a $248 \mathrm{~nm}, 26 \mathrm{~ns}, \mathrm{KrF}$ excimer laser at close to normal incidence, was used to ablate a rotating silver target in a vacuum chamber at $5 \times 10^{-5}$ mbar. The spot size was $3 \times 1.5 \mathrm{~mm}^{2}$ and the laser fluence was 1.5 $\mathrm{J} \mathrm{cm}^{-2}$. A planar Langmuir probe with dimensions $L=13$ $\mathrm{mm}$ and $W=3 \mathrm{~mm}$ was mounted so as to rotate on a circle centered on, and facing, the ablation spot. Both theory and experiment show that for a planar probe facing the plasma flow and at negative voltages high enough to prevent the electrons with the highest thermal energies in the plasma from reaching the probe, the detected ionic current saturates. ${ }^{12}$ This current is proportional to the ion density and the ion flow velocity, $v_{i}$, through the following expression:

$$
I_{i}=e v_{i} A n_{i},
$$

where $e$ is the electron charge and $A$ is the collecting area of the probe. The above equation assumes that only singly charged ions are present. Neglecting the acceleration time of the ablated plume, $v_{i}$ is given by the target-probe distance divided by the TOF. The ion current density $J(t)$ also yields the ion energy distribution via the following relation:

$$
d F / d E=J(t) \times t^{3} / e m_{i} z^{2} .
$$

\section{RESULTS AND DISCUSSION}

Figure 4(a) shows the ion current when the probe is placed $9.5 \mathrm{~cm}$ along the target normal and oriented normal to the plasma flow. The plume front arrives to the probe at $t_{f}=2.5 \mu \mathrm{s}$, it reaches a maximum that corresponds to the time of maximum ion flux at $6.5 \mu \mathrm{s}$ and then falls as the plume continues to expand beyond the probe. Figure 4(a) also shows the corrected ion density derived from the probe

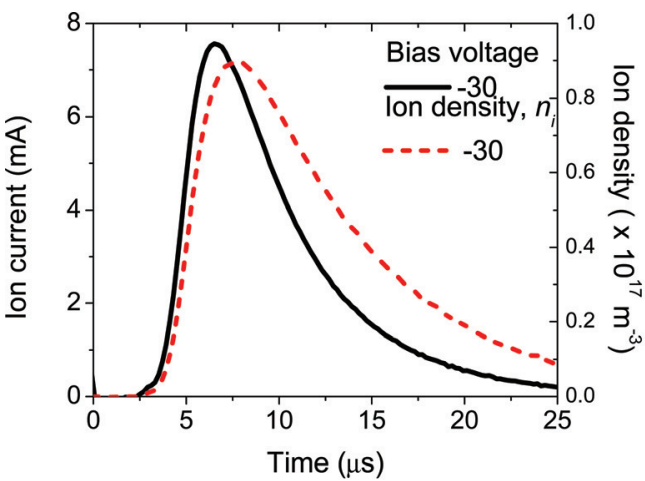

(a)

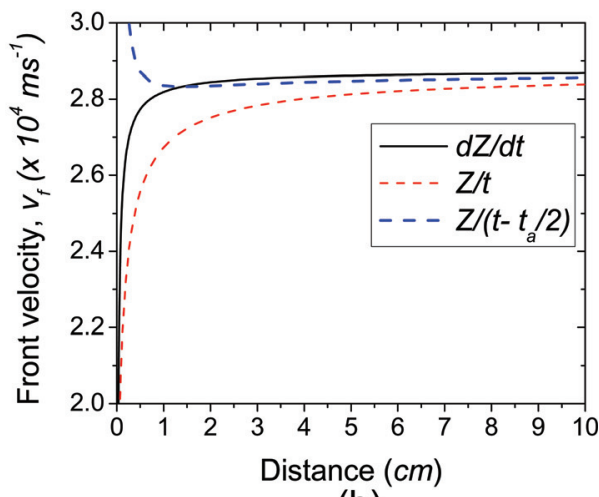

(b)
FIG. 4. (Color online) (a) Ion current and density at $z=9.5 \mathrm{~cm}$ vs time (b). The ion front velocity determined from the Anisimov model using the plume front position $(Z / t)$ compared to the instantaneous velocity $d Z / d t$, also determined from the Anisimov model. 


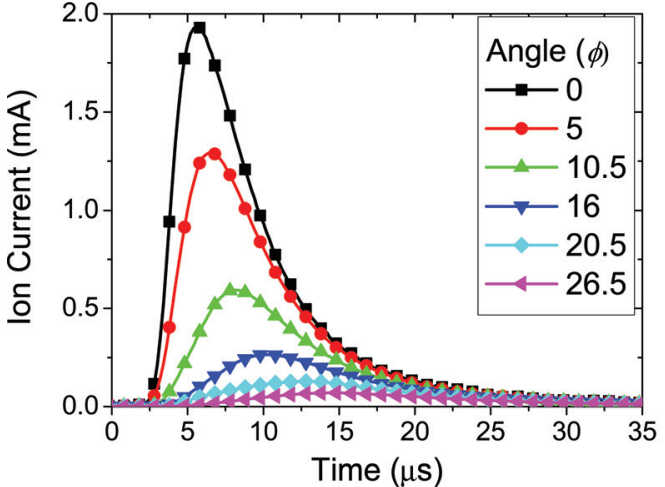

(a)

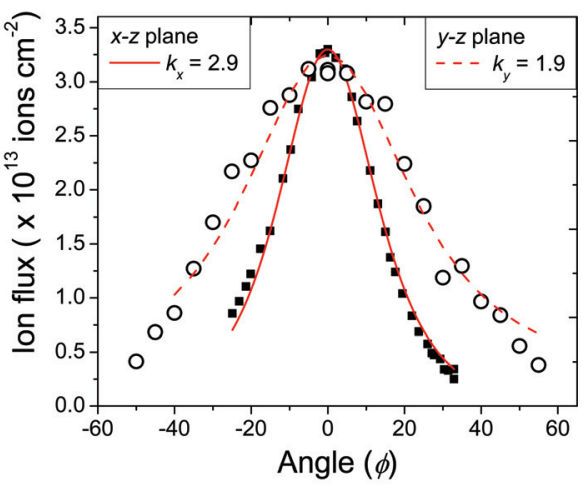

(b)
FIG. 5. (Color online) (a) Ion probe signals for various angles relative to the target normal. (b) The angular distribution of the ion fluence obtained by integrating the ion signals in both the $x-z$ and $y-z$ planes at a distance of $4.1 \mathrm{~cm}$. signal obtained according to Eq. (19) and using the ion velocity, $v_{i}$, as described below.

The ion flow velocity is probably the simplest plasma parameter to determine using a Langmuir probe. The flow velocity is determined from data like that in Fig. 4 (a), by using the equation $v_{i}=z / t$. However as $t=0$ corresponds to the time the laser hits the target, this disregards the time taken for the ablation, plume formation and the initial acceleration of the plasma. Figure 4 (b) shows the ion front velocity from the Anisimov model using the plume front position, $(Z / t)$, and the instantaneous velocity, $d Z / d t$, also determined from the Anisimov model. The discrepancy, while small, is easily corrected. We can approximate the plume front as accelerating from rest for a time, $t_{a}$, after which it reaches a constant velocity, $v_{f}$. The distance traveled is then, $s=z$ $=v_{f}\left(t-t_{a} / 2\right)$ and is also shown. From Fig. 4(b) we can calculate $t_{a}$ for each distance, $z$. The average acceleration time was determined to be approximately $50 \mathrm{~ns}$, which is small compared to the time taken for the plume to reach the probe.

By moving the probe around the target at a fixed distance, the shape of the plume can be obtained. Figure 5(a) shows the angular variation of ion probe signals for laser ablation of silver recorded with an ion probe which can be moved on a radius of $4.1 \mathrm{~cm}$ about the ablation spot on the

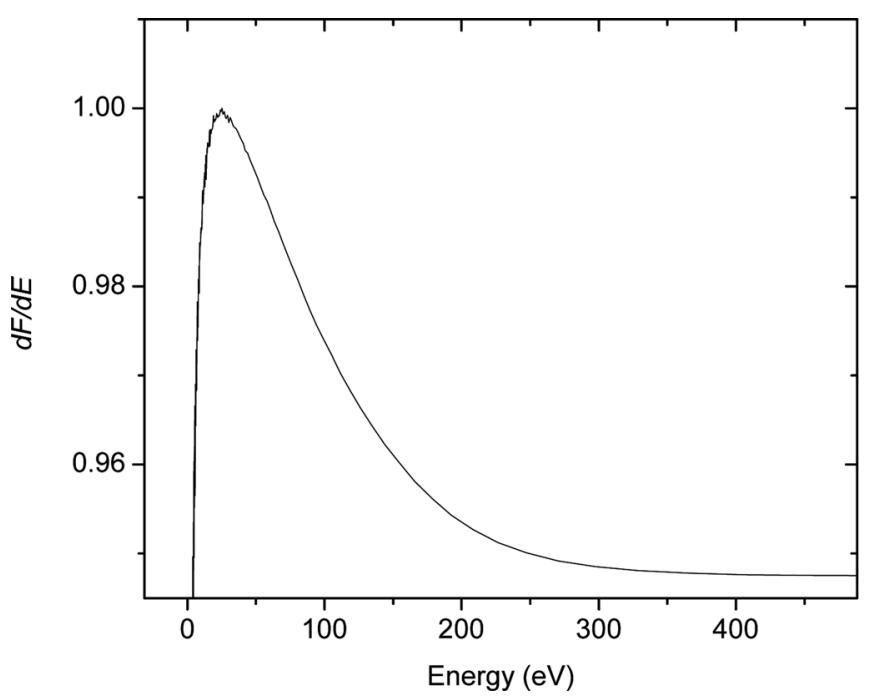

FIG. 6. Normalized ion energy distribution corresponding to the ion current signal from Fig. 5(a). target. The signal amplitude is maximized and the TOF minimized in the direction normal to the target according to Eq. (12). The plasma front arrives at $2.5 \mu$ s which corresponds to $300 \mathrm{eV}$. From the measured plume front expansion velocity we can derive a value for $E_{p} / M_{p}$ from Eq. (14). The initial value of $E_{p} / M_{p}$ corresponds to $5.3 \times 10^{7} \mathrm{~J} \mathrm{~kg}^{-1}$, or $67 \mathrm{eV}$ per atom or ion. The two orthogonal angular distributions of the ion fluence are found by integrating the TOF signals. The distributions in the $x-z$ and $y-z$ planes are shown in Fig. 5(b) and are fitted to Eq. (15) using $k_{x}=2.9$ and $k_{y}=1.9$. The ion kinetic energy distribution in the plasma flow in a particular direction can be obtained from the corresponding ion signal using Eq. (20). Figure 6 show the energy distribution for the flow normal to the target surface; the mean ion energy is $100 \mathrm{eV}$, and the most probable energy is $30 \mathrm{eV}$. We have determined that the mass of the plume is approximately $60 \mathrm{ng}$ which gives a plume energy of $3 \mathrm{~mJ}$. The incident laser energy was approximately $25 \mathrm{~mJ}$ per pulse.

It is possible to use the experimental values of $k$ together with the Anisimov model to determine an initial plasma thickness $Z_{0}$. Fig. 7 shows the evolution of plume shape for an initial spot size of $1 \times 0.5 \mathrm{~mm}, \gamma=1.25$ and for $k$-values close to the experiment. $Z_{0}$ corresponds to approximately 65 $\mu \mathrm{m}$. Additionally, the plots in Fig. 7 set the time scale for the evolution of plume shape. At $\tau=2(\sim 60 \mathrm{~ns})$ the $y$ semiaxis

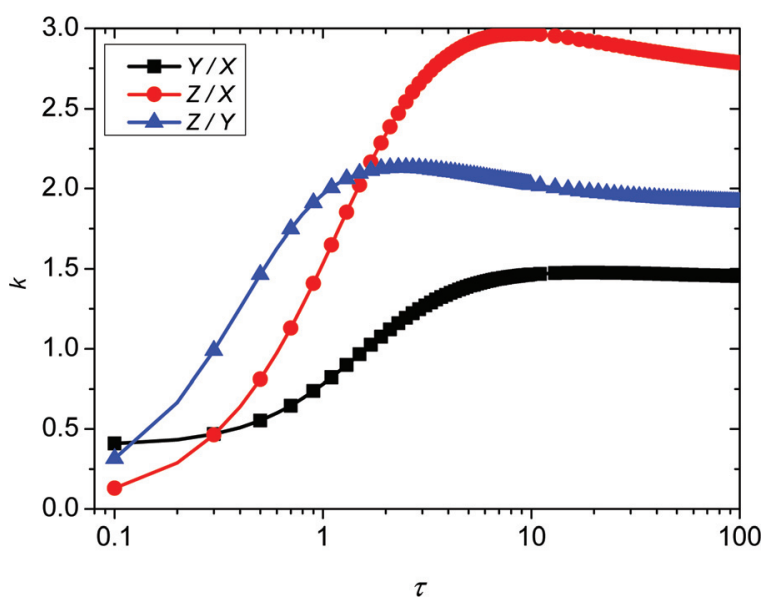

FIG. 7. (Color online) Calculated variation of the plume aspect ratios for a plume with initial spot dimensions: $X_{0}=0.5 \mathrm{~mm}, Y_{0}=0.25 \mathrm{~mm}$, and $Z_{0}=65 \mu \mathrm{m}$. 


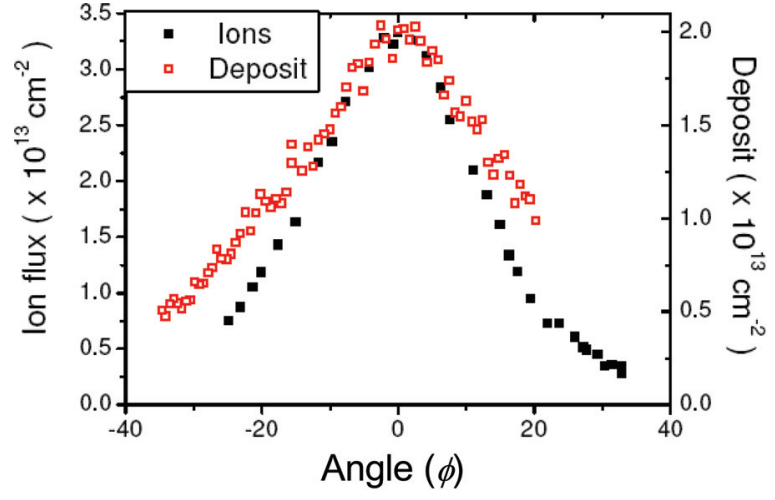

FIG. 8. (Color online) Angular distributions in the $x$-z plane of the integrated ion flux and net deposition on a circular surface of radius $4.1 \mathrm{~cm}$ centered on the ablation spot.

$(Y)$ becomes larger than $x$ semiaxis $(X)$, and at $\tau \sim 3(\sim 180$ ns) the plume elongation is nearly fully established.

Finally we compared the angular distributions of the ion fluence and the net deposition. A rotating silver target was ablated using 40000 shots at $2 \mathrm{~J} \mathrm{~cm}^{-2}$ and the ablated material deposited on a transparent film. By measuring the optical transmission through the film, before and after the deposition, the thickness and angular distribution of material was calculated and is shown in Fig. 8. Fitting the angular distributions in the same way as before yielded $k_{x}=1.9$ and $k_{y}=1.2$ for the deposit. These values can be compared with $k_{x}=2.9$ and $k_{y}=1.9$ for the ion fluence distribution, showing that the ions have a somewhat narrower distribution. The total number of atoms, $N$, collected on a hemispherical surface of radius $z_{p}$ centered on the ablation spot can also be estimated from the Anisimov model using the equation

$$
N=\frac{2 \pi z_{p}^{2} f(0)}{k_{x} k_{y}},
$$

where $f(0)$ is the integrated flux at $0^{\circ}$. The thickest part of the film was determined to be $45 \mathrm{~nm}$, which equates to $f(0)=6.5 \times 10^{12}$ particles $\mathrm{cm}^{-2}$ pulse ${ }^{-1}$. Using the measured $k$-values, the yield was estimated to be $5.8 \times 10^{14}$ particles per pulse. Similarly from the ion probe measurements the yield was estimated to be $6.2 \times 10^{14}$ ions per pulse.

The mass loss and corresponding ablation depth was determined by weighing the target before and after ablation for $60 \mathrm{~min}$. at $10 \mathrm{~Hz}$. The ablation rates for a fluence of 1.5 and $2 \mathrm{~J} \mathrm{~cm}^{-2}$ were determined to be 65 and $200 \mathrm{ng}$ per shot, respectively. The corresponding ablation depths are 5 and 15 $\mathrm{nm}$. A mass of $200 \mathrm{ng}$ of silver corresponds to $1.1 \times 10^{15}$ atoms. Comparing the ion yield to the mass loss, the ion fraction is then approximately 0.56 . Table I shows the values for number of atoms ablated, ion yield and net deposition for ablation of silver at $2 \mathrm{~J} \mathrm{~cm}^{-2}$ on a $0.5 \mathrm{~mm}^{2}$ laser spot. It can be seen that the overall ion faction is $\sim 56 \%$. It can also be seen that the net deposition corresponds to about $50 \%$ of the atoms ablated, indicating self-sputtering of the growing film
TABLE I. A summary of the results for the ablation of silver at $2 \mathrm{~J} \mathrm{~cm}^{-2}$.

\begin{tabular}{lccc}
\hline \hline Atoms ablated & Ion yield & Ion fraction & Atoms deposited \\
\hline $1.1 \times 10^{15}$ & $6.2 \times 10^{14}$ & 0.56 & $5.8 \times 10^{14}$ \\
\hline \hline
\end{tabular}

due to recoil of energetic ions and neutrals is significant. This is not surprising in view of the ion energy spectrum shown in Fig. 6.

\section{CONCLUSIONS}

In conclusion, we have shown that the adiabatic gasdynamic model of laser ablation plume expansion developed by Anisimov et al. can also be used when the plume is ionized. We have described how a simple planar Langmuir probe can be used to measure various aspects of the expansion of the plasma part of the ablation plume of a silver target irradiated with $\mathrm{ns}$ pulses at $1.5-2 \mathrm{~J} \mathrm{~cm}^{-2}$. We have also shown that the Langmuir probe can be used to obtain a fairly comprehensive description of the expansion dynamics of the ionized part of the ablation plume, including plume shape and ion energy distribution. There is a good agreement between the ablated mass and the net deposition per pulse. We also estimate the fraction of the initial laser energy which is absorbed in the ablated material and drives the plume expansion.

\section{ACKNOWLEDGMENTS}

The research was funded by Enterprise Ireland under research Grant No. SC/02/363.

${ }^{1}$ A. Vertes, R. Gijbels, and F. Adams, Laser Ionization Mass Analysis, Vol. 124 of Chemical Analysis Series (Wiley, New York, 1993).

${ }^{2}$ Pulsed Laser Deposition of Thin Films: Applications- Led Growth of Functional Materials, edited by R.W. Eason (Wiley, New York, 2006).

${ }^{3}$ T. Donnelly, B. Doggett, and J. G. Lunney, Appl. Surf. Sci. 252(13), 4445 (2006).

${ }^{4}$ J. G. Lunney and R. Jordan, Appl. Surf. Sci. 127, 941 (1998).

${ }^{5}$ T. E. Itina, J. Hermann, P. Delaporte, and M. Sentis, Appl. Surf. Sci. 208, 27 (2003).

${ }^{6}$ S. I. Anisimov, B. S. Luk'yanchuk, and A. Luches, Appl. Surf. Sci. 96, 24 (1996).

${ }^{7}$ R. K. Singh and J. Narayan, Phys. Rev. B 41(13), 8843 (1990).

${ }^{8}$ L. Spitzer, Physics of Fully Ionized Gases, 2nd ed., Tracts on Physics and Astronomy No. 3 (Interscience, New York, 1962).

${ }^{9}$ D. T. Attwood, Soft X-rays and Extreme Ultraviolet Radiation: Principles and Applications (Cambridge University Press, Cambridge, 2000).

${ }^{10}$ T. N. Hansen, J. Schou, and J. G. Lunney, Appl. Phys. A: Mater. Sci. Process. 69, 601 (1999).

${ }^{11}$ B. Toftmann, J. Schou, T. N. Hansen, and J. G. Lunney, Phys. Rev. Lett. 84(17), 3998 (2000).

${ }^{12}$ B. Doggett and J. G. Lunney, J. Appl. Phys. 105, 033306 (2009).

${ }^{13}$ S. Amoruso, R. Bruzzese, N. Spinelli, and R. Velotta, J. Phys. B 32, R131 (1999).

${ }^{14}$ D. W. Koopman, Phys. Fluids. 14, 1707 (1971).

${ }^{15}$ R. J. von Gutfeld and R. W. Dreyfus, Appl. Phys. Lett. 54, 1212 (1989).

${ }^{16}$ Ya. B. Zeldovich and Yu. P. Raizer, Physics of Shock Waves and HighTemperature Phenomena (Dover Publications, New York, 2002). 